\title{
ANÁLISIS DEL MARCO TRIBUTARIO DE LOS PAISES MIEMBROS DEL MILA Y SU EFECTO EN LA NEGOCIACION DE ACCIONES ENTRE PAISES'
}

\author{
Analysis of the MILA's tax policies and its effect on the negotiation of actions \\ between countries
}

\author{
Diego Arley Gil Solano² \\ Institución Universitaria Politécnico Grancolombiano \\ Bogotá, Colombia
}

\begin{abstract}
Resumen
El mercado integrado latinoamericano (MILA), conformado por las bolsas de valores de Chile, México, Perú y Colombia, es una de las iniciativas más significativas en cuanto a la integración regional a través de un mercado bursátil que apunta no sólo a convertirse en el más grande en América latina, sino además en una plaza sólida y atractiva en la que es posible negociar títulos de renta variable, entre otros productos.

Sin embargo, esta integración no se ha dado de la manera esperada debido a las asimetrías regulatorias existentes entre los mercados que lo integran. El propósito de esta investigación es analizar las asimetrías tributarias existentes entre los países miembros del MILA y si estas tienen un efecto en la integración de los mercados; para la investigación se realiza un comparativo de las tarifas de los impuestos de los diferentes países asociados a la compra y venta de acciones en personas jurídicas, con base en esta información, se desarrolla un modelo en Excel con el cual se ejemplifica una inversión hipotética en los distintos mercados que conforman el MILA.
\end{abstract}

Palabras clave: Mercado Integrado Latinoamericano (MILA), Marco tributario, impuestos, inversionista, acciones.

\footnotetext{
${ }^{1}$ Cite este artículo como: Gil-Solano, D. (2020). Análisis del marco tributario de los países miembros del MILA y su efecto en la negociación de acciones entre países, Revista Punto de Vista, 11 (17), pp. 31-48.

${ }^{2}$ Magíster en Negocios Internacionales de la Institución Universitaria Politécnico Grancolombiano. Director de operaciones Backoffice ICAP. Correo electrónico: dgilsolano@set-icap.co.
} 


\begin{abstract}
The Integrated Latin American Market (In spanish, Mercado Integrado Latinoamericano, MILA) is a stock Exchange project formed by the local exchanges of Chile, Mexico, Peru and Colombia. It was conceived to integrate these exchanges thus becoming the region's largest capital market. Such integration, nonetheless, has not evolved as expected due to asymmetries present among the members' local tax regulations. The purpose of this research document is to analyze such asymmetries, as well as the way they impact market integration. For this purpose, a comparison analysis is carried out, of their local tax regulation applicable to purchase and sale of financial instruments.
\end{abstract}

Key words:

Latin America integrated market (MILA), tax policy, stock market, investment.

Recepción:

24.07.2020

Aceptación:

07.10 .2020

\title{
INTRODUCCIÓN
}

El MILA busca fomentar el crecimiento de la actividad bursátil, ofreciendo una infraestructura eficiente y competitiva con estándares internacionales, una mejor y mayor exposición de sus mercados y una mayor oferta de productos y oportunidades para los inversionistas locales y extranjeros. Para el caso colombiano, el MILA puede dar a conocer más las empresas listadas en la bolsa de valores, para así fortalecer el mercado bursátil local, es así como el MILA podría ser un medio para el progreso del mercado bursátil colombiano (Lancheros, 2017).

Sin embargo, esta integración no ha alcanzado el potencial que se había proyectado, el MILA es un mercado bursátil que no es suficientemente conocido, por lo cual para llegar a un mayor número de inversionistas es necesario mayor promoción de la plataforma y de su funcionamiento, ya que el MILA empezó a operar desde el año 2011 y aunque ha presentado un crecimiento en el valor negociación año a año, para alcanzar mayores niveles de negociación en el mercado, se debe ampliar la información al inversionista de su funcionamiento y como puede operar de una manera sencilla y práctica, así como también una información clara y concisa de la normatividad sobre los impuestos en los que puede incurrir en la negociación de un activo financiero de las empresas de cada país integrante del MILA.

Una de las causas de que el mercado no esté alcanzando los resultados estimados, es la asimetría regulatoria existente entre los países miembros, esto hace que ciertos productos no puedan salir al mercado MILA y en otros casos limita la posibilidad de que los inversionistas estén con las mismas condiciones al invertir en cualquiera de los países miembros (Almazán, 2015).

Por este motivo, es importante realizar un análisis de la carga tributaria que los inversionistas tienen al operar en cada uno de los países miembros del MILA, específicamente la asociada con la compra y venta de acciones de 
personas jurídicas; para ello la investigación realizada se hace desde dos enfoques, el primero de ellos mediante un análisis comparado de los costos tributarios asociados con las transacciones en acciones y el segundo, es un enfoque numérico en el cual mediante un caso hipotético se evalúa en términos cuantitativos la carga tributaria asumida por un inversionista que opere en cada uno de los mercados analizados. El ejercicio simulado se construye usando variables como la tasa de cambio, inversión inicial, comisión hipotética sobre transacciones de compra y venta de acciones, periodo de la inversión, valor de la acción, porcentaje de rentabilidad esperada, los dividendos a repartir para el ejercicio y el monto de la inversión que se supone no supera el $7 \%$ de las acciones emitidas por una misma empresa.

Los resultados indican que la mayor rentabilidad en cuanto a lo que se refiere a dividendos y ganancias de capital se encuentran en los países de Perú y México, ya que poseen unas bajas tasas de impuestos en comparación con Colombia y Chile. Sin embargo, en cuanto a los volúmenes de negociación reportados por el MILA, Perú posee bajos volúmenes de negociación, por lo cual las tasas de impuestos a inversionistas del exterior sobre personas jurídicas en cuanto a ganancias de capital y dividendos no son acordes con los volúmenes de negociación de acciones que tienen los países del MILA.

\section{Contextualización del MILA}

El MILA es una plataforma bursátil virtual que empezó a operar a partir del año 2011, fue creada sin fusión corporativa para la integración de las bolsas de valores de los países de Chile, Perú, Colombia y México. La iniciativa surge con el objetivo de convertirse en el mercado de capitales más grande de Latinoamérica (MILA Mercado Integrado Latinoamericano, s.f.) a través de las transacciones de las acciones de las empresas que se encuentran listadas en las bolsas de valores de los países que lo integran. El 30 de mayo del año 2011 se inician las operaciones del MILA. La primera fase del proyecto, la integración de las bolsas de valores se da a través de un enrutamiento intermediado, el cual radica en que funcionarios en cada bolsa son los responsables de las órdenes de compra y venta. Posteriormente se da la segunda fase que tendría por objetivos el acceso directo de intermediarios, la estandarización de reglas de negociación, la definición de un modelo de compensación y liquidación transfronterizo (Mila, s.f.)

El mercado integrado latinoamericano en un principio brinda un portafolio de inversión en renta variable, renta fija, derivados y otros productos sobre más de 600 empresas de estos cuatro países. Más de 60 intermediarios interconectados, así como la liquidación y compensación, la custodia de valores se encuentra en los mercados de origen con completa interconexión entre depósitos. El objetivo de los países de Colombia, México, Perú y Chile es la creación de un mercado integrado de valores que sea atractivo para diferentes tipos de inversionistas extranjeros y nacionales, para así ser un mercado más sólido y robusto, donde los inversionistas puedan diversificar sus inversiones de acuerdo con las fortalezas que tienen los países integrantes del MILA en diversos sectores económicos.

\section{Evolución del MILA}

Para dar cuenta de la evolución del proceso de integración se mostrarán los diferentes datos numéricos, mediante cuadros y estadísticas de cifras relevantes que sustentan el comportamiento y avance del MILA, posteriormente, se realiza un análisis de las imperfecciones del mercado desde la óptica tributaria.

La capitalización bursátil se entiende básicamente como una medida económica que muestra el valor de una empresa de acuerdo con el precio del mercado. También se puede entender como el valor de todas las acciones de una empresa que está cotizando en la bolsa de valores. Por esta razón el tenedor de una acción, es también propietario de una parte de la empresa, en definitiva, la capitalización bursátil de una empresa, es su valor al precio del mercado (Academia de Inversión, 2013) 


\begin{tabular}{|c|c|c|c|c|c|c|c|c|c|c|}
\hline & 2015 & 2016 & 2017 & $\begin{array}{l}\text { V absoluta } \\
2015-2016\end{array}$ & $\begin{array}{l}\text { V absoluta } \\
2016-2017\end{array}$ & $\begin{array}{l}\text { V Relativa } \\
2015-2016\end{array}$ & $\begin{array}{l}\text { V Relativa } \\
2016-2017\end{array}$ & $\begin{array}{c}\text { Part } \\
\%\end{array}$ & $\begin{array}{c}\text { Part } \\
\%\end{array}$ & $\begin{array}{c}\text { Part } \\
\%\end{array}$ \\
\hline Chile & 190.625 & 209.857 & 296.286 & 19.232 & 86.429 & $10 \%$ & $41 \%$ & $25 \%$ & $27 \%$ & $32 \%$ \\
\hline Colombia & 88.488 & 103.770 & 121.932 & 15.282 & 18.162 & $17 \%$ & $18 \%$ & $11 \%$ & $13 \%$ & $13 \%$ \\
\hline Perú & 90.657 & 124.044 & 98.684 & 33.387 & 25.360 & $37 \%$ & $-20 \%$ & $12 \%$ & $16 \%$ & $11 \%$ \\
\hline México & 401.033 & 351.687 & 415.820 & 49.346 & 64.133 & $-12 \%$ & $18 \%$ & $52 \%$ & $45 \%$ & $45 \%$ \\
\hline Total & 770.803 & 789.358 & 932.722 & 18.555 & $143 \cdot 364$ & $2,4 \%$ & $18,2 \%$ & $100 \%$ & $100 \%$ & $100 \%$ \\
\hline
\end{tabular}

Tabla 1 Capitalización Bursátil MILA. Fuente: (MILA Mercado Integrado Latinoamericano, 2018)

De acuerdo con la información contenida en la tabla 1 se observa que la capitalización del MILA de los años 2015, 2016 y 2017 se incrementa pues entre 2015 y 2016 varió 2.4\% y entre 2016 y 2017 creció 18\%. El país que posee una mayor capitalización bursátil es México con un 45\% de la capitalización del MILA para el año 2016 y 2017, Perú es el país con menor capitalización con un 16\% y 11\% para el 2016 y 2017 respectivamente, Colombia se ha mantenido estable con el 13\% para el 2016 y 2017 y con un valor de capitalización para el 2017 de \$121.932 millones de dólares.

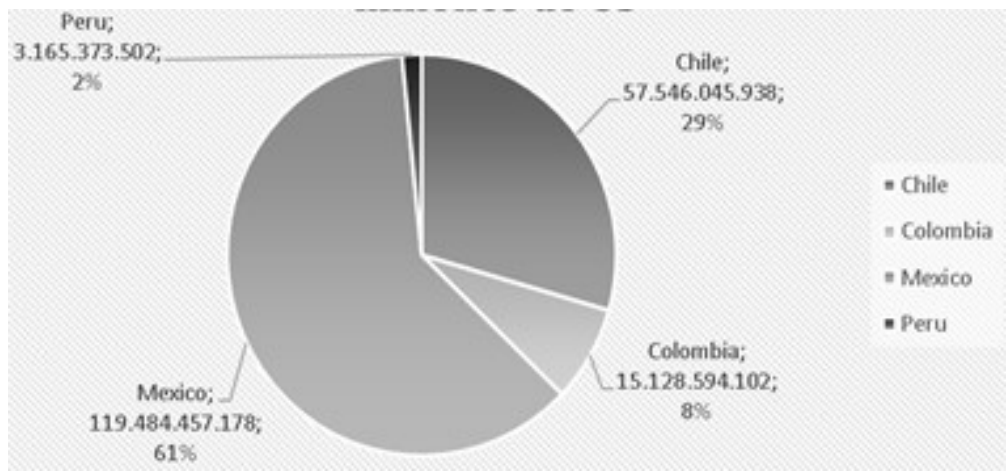

Figura 1 Volumen Negociado en el MILA 2018 en millones de US. Fuente: Elaboración propia basado en datos de http://mercadomila.com/datosdel-mercado/volumenes/

Como se observa en la Figura 1, el mayor volumen de negociación en el año 2018 está dado en las empresas del país de México representando un 61\% del volumen negociado en el MILA, seguido en segundo lugar por Chile con un $29 \%$, en tercer lugar, Colombia con un $8 \%$ y por ultimo Perú con un $2 \%$, esto evidencia que todavía Colombia tiene mucho por mejorar, ya que su participación es la tercera después de México y Chile en cuanto a volúmenes de negociación del MILA en este año.

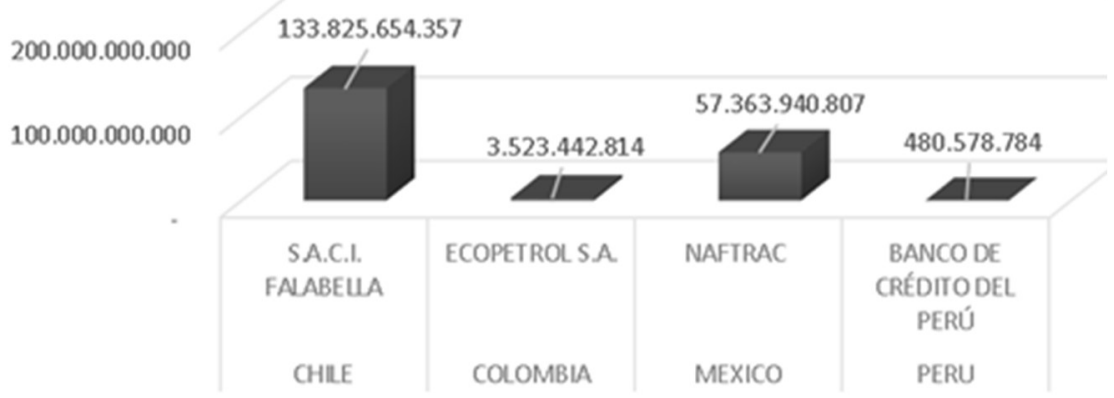

Figura 1 Instrumentos más Negociados por País en el MILA. Fuente: Elaboración propia basado en los datos de http://mercadomila.com/?s=capitalizacion\&lang=es 
De acuerdo con la Figura 2, se presentan las acciones de las empresas con mayor volumen de negociación durante el año 2018, donde se observan los principales emisores del mercado integrado, para el caso de Chile Falabella fue la acción más negociada, con los mayores volúmenes tranzados especialmente en los últimos meses del año, en segundo lugar está la empresa Naf Trac de México, en tercer lugar se encuentra la empresa Ecopetrol, la empresa más grande del mercado colombiano medida por capitalización bursátil, y la cual registró un volumen de negociación en el MILA de 3.523.442.814 dólares; finalmente se encuentra el Banco de Crédito del Perú, con un monto negociado de 480.578.784 dólares.

\begin{tabular}{|lr|}
\hline & Emisores en el MILA - Diciembre 2018 \\
\hline Chile & 205 \\
\hline Colombia & 68 \\
\hline México & 140 \\
\hline Perú & 225 \\
\hline Total Emisores MILA & Tabla 2 Emisores mercado MILA. Fuente: (MILA Mercado Integrado Latinoamericano, 2018) \\
\hline
\end{tabular}

En la la tabla 2 se encuentra que el mercado de valores colombiano es el mas pequeño en lo que respecta al numero de emisores que lo conforman. En contraste, el mercado de mayor tamaño por numero de emisores es el mercado peruano, seguido de cerca por el mercado chileno. Llama la atención que para los mercados analizados el número de emisores no es un indicador de tamaño de la negociación, debido a que el mercado de Perú es el que tiene mayor número de emisores, sin embargo, es el que cuenta con menores volúmenes de negociación.

Si bien es cierto que las estadísticas muestran avances importantes en la integración de los mercados, de acuerdo con Lancheros (2017) se debe tener una mayor unión de los eslabones de la cadena para que el MILA alcance su potencial y es necesaria una mayor promoción del mercado integrado entre los inversionistas, ofrecer incentivos en costos transaccionales y unificación de la información tributaria para que cualquier inversor este bajo las mismas condiciones en cuanto a carga tributaria, independientemente en qué país del mercado MILA en el que invierta, la plataforma todavía sigue siendo desconocida para muchos. El mercado necesita para su desarrollo, un mayor número de inversionistas, ya que de acuerdo con Lancheros (2017) Las ganancias obtenidas por un inversor en el mercado aumentan con el número de participantes, fortaleciendo el mercado e incrementando la liquidez de un título, generando mayor número de transacciones. Por su parte, Colombia es el país que presenta mayores dificultades en cuanto a presentación de información financiera bajo estándares internaciones como NIIF y NIC.

\section{La teoría y la importancia del contexto tributario}

Según Modigliani \& Merton (1958) una de las mayores imperfecciones de los mercados financieros está relacionada con la existencia de impuestos, así como costos transaccionales y la asimetría informativa, entre otros. Para el año de 1963 estos autores propusieron el Teorema de Modigliani-Miller, el cual es la base de las teorías modernas sobre la estructura del capital, el teorema indica que independientemente del mercado, el valor de una empresa no se ve afectado por la forma de financiación que tiene la empresa, para su operación, así su financiación del capital provenga de medios como la emisión de acciones o de algún préstamo, tampoco depende de la política de repartición de dividendos de la compañía. Sin embargo, según el teorema, con la aparición de los impuestos corporativos se descompone la estructura de financiación ya que el coste de las deudas se reduce porque es un gasto que se paga antes del impuesto sobre beneficios (Rivera, 2002). 
Por su parte Fama propuso su teoría sobre los mercados eficientes, de acuerdo con la cual precios de las acciones y demás valores que se comercializan en los mercados de capitales expresan continuamente toda la información disponible a través de sus precios. Este fenómeno es provocado por los mismos inversores, ya que es un resultado de su comportamiento maximizador sobre la base de su conocimiento y sus creencias que les sirven de base para sus proyecciones en el futuro de cuánto puede subir o bajar el precio de un producto financiero. Así los inversionistas al realizar operaciones de mercado hacen que los precios se muevan hasta alcanzar que los retornos esperados ajustados por riesgos sean iguales para todos los activos (Uribe \& Ulloa, 2011)

Cualquier movimiento que se dé en el precio de un activo se debe a eventos no conocidos de antemano que rápidamente son incorporados en los nuevos precios. Por lo cual nadie le puede ganar al mercado. La información que se da en el mercado puede influir en el alza o baja de un producto financiero: noticias con fines políticos, proyecciones de crecimiento alcanzadas o no alcanzadas, alianzas comerciales entre empresas o investigaciones a empresas por malos manejos, todo este tipo de información es de gran importancia para saber si una acción por ejemplo, va a subir o va a bajar, esto es otro factor importante, que deben tener presente los inversionistas, para obtener un buen retorno de sus inversiones en activos financieros (Cabieses, 2013).

Markowitz en su artículo Portfolio Selection de 1952 (Franco, Avendaño, \& Barbutin, 2011) propuso que mediante la observación y análisis, sumado con la experiencia y expectativas de inversiones futuras, se podían crear portafolios de inversión, esta idea es aplicada por muchos expertos en el para el análisis de los activos financieros a invertir, por ejemplo, para armar un portafolio de acciones del MILA, en las cuales para crear mercado y dinamismo, el inversionista debe realizar una análisis de los históricos de la acción y gráficos e indicadores del movimiento del precio de las acciones a través de la observación, aplicando su experiencia y teniendo en cuenta las expectativas del mercado y proyecciones de las empresas, en sus planes de expansión y crecimiento y qué tan alcanzables son según lo proyectado (Banco Nacional de Mexico, s.f.).

Tomando lo expuesto por los autores citados anteriormente para un inversionista que comercializa acciones de cualquiera de las empresas que se encuentran en el mercado MILA, es importante conocer las fechas en las que se reparten dividendos, así como la estructura de financiamiento de la empresa y sus planes de expansión, la observación del mercado, la experiencia que tiene el inversionista y las proyecciones de las empresas que emiten las acciones y el rendimiento de sus acciones, las tasas de impuestos sobre ganancias por dividendos y capital, así como la carga tributaria que tiene la empresa en el país en el que se realiza la inversión, entre otros factores que debe considerar un inversionista para realizar una inversión y que son muy importantes y que hacen la diferencia entre la posible ganancias o perdida que tendrá el inversionista.

En cuanto al efecto de los impuestos, en gran parte de los sistemas occidentales los ingresos recibidos por los inversores bajo la forma de dividendos son penalizados fiscalmente sobre las ganancias de capital ya que éstas últimas están sometidas a tipos menores. Los inversionistas deberán analizar muy bien las empresas que tengan acciones con una elevada razón de reparto de dividendos ya que, los inversores al ver esto desde la rentabilidad después de impuestos exigirán, para un riesgo dado, rentabilidades antes de impuestos superiores (Universidad de Oviedo, s.f.)

Añadido a esto, es importante analizar las ventajas y desventajas fiscales, que ofrecen diferentes alternativas como la retención de beneficios, recompra de acciones frente al reparto de dividendos. Un ejemplo de una ventaja fiscal es en la retención de beneficios por poder diferirse el pago de impuestos hasta el momento de la venta de acciones. Así, el inversor paga los mismos impuestos en ambas situaciones, aunque en distintos momentos de tiempo, por lo que los accionistas preferirán ganancias de capital al permitirles diferir el pago de impuestos.

Otro ejemplo se da cuando los inversionistas de una tasa de renta elevada, con dividendos gravados a altos tipos marginales prefieren un escaso o nulo reparto de dividendos, con la esperanza de obtener mayores ganancias de 
capital. Para los inversionistas también es muy importante si las acciones en las que invierten son de empresas con altos o bajos repartos de dividendos, ya que en acciones de empresas con bajos repartos de dividendos las deberían adquirir en mayor medida inversionistas con elevados tipos impositivos marginales, por otro lado, las acciones de empresas con elevados dividendos deberían ser adquiridas por inversores con bajos tipos marginales (Universidad de Oviedo, s.f.)

En los últimos años, la normatividad tributaria de los países miembros del MILA ha tenido diversos cambios, estos han impactado directamente a la negociación de acciones en los mercados que lo conforman, adicionalmente, los países miembros han firmado acuerdos de no doble tributación con el propósito de fomentar el intercambio de flujos de capital. A continuación, se hace un recuento de los principales ajustes tributarios realizados en los países que integran el MILA en los últimos años.

\section{Chile}

En Chile se realizan modificaciones al sistema de tributación de renta, se realizan ajustes en el IVA principalmente en operaciones comerciales con inmuebles, se realizan mejoras en la vigencia de la norma general anti-elusión, se desarrollan beneficios tributarios a la inversión y establecen medidas para el impulso a la productividad la cual se basa impuestos directos pagados en el exterior para exportadores de servicios, evitando la doble tributación (Ley No 20.956, 2016).

\section{Colombia}

En Colombia se han realizado Reformas tributarias estructurales: Con el impuesto a la renta de personas naturales: a través de determinación cedular, se incrementa la tarifa máxima para rentas no laborales y de capital, se gravan dividendos con tasas progresivas. Así mismo, se eliminan los impuestos del IMAS e IMAN. Para el Impuesto sobre la renta de las empresas: Se unifica la tasa en 34\% para 2017 y 33\% a partir de 2018 más sobretasa de 6\% (2017) y 4\% (2018). Se elimina el impuesto a la riqueza y CREE (Ley 1819, 2016)

Se establece un aumento en la tasa general del IVA (de 16 al 19\%), se grava con tasa de $5 \%$ la primera venta de viviendas nuevas de alto valor y tasa reducida del $5 \%$ para ciertos productos. Se crea el Monotributo y se establece como permanente el gravamen a los movimientos financieros; además, se modifica impuestos territoriales y se establecen incentivos tributarios para las zonas más afectadas por el conflicto armado (Ley 1819, 2016).

\section{México}

Se incentivan los beneficios fiscales a la inversión en equipos de alimentación de vehículos eléctricos (tasa del o por ciento); tasa del o por ciento a la exportación de servicios de tecnologías de información y en proyectos de promoción del deporte de alto rendimiento. Se determina un sistema de tributación opcional del ISR para las PYMES (Ley General de Sociedades Mercantiles, 2018)

\section{Perú}

Se establece una disminución de la tasa de retención por ganancias de capital enfocado a la enajenación de inmuebles de $30 \%$ a $5 \%$. Se establece un incremento de la tasa del ISR para empresas del $28 \%$ a $29.5 \%$. Se disminuye la tasa de retención a los dividendos: de 6,8 a 5\%. Por último, se establece un régimen tributario especial para las micro y pequeñas empresas (RMT) Fraccionamiento de deudas tributarias de personas naturales y las Pymes (Decreto Supremo N $133-2013-E F, 2016)$ 
Convenios de no doble tributación

\begin{tabular}{|c|c|c|c|c|}
\hline País & Colombia & Perú & Chile & México \\
\hline Colombia & $x X$ & Decisión 578 de 2004 CAN & $\begin{array}{l}\text { LEY } 1261 \text { de } 2008 \text { (Col) y Dec Supr No } \\
232 \text { de2009 (Chi) }\end{array}$ & Ley 1568 del 2012 \\
\hline Perú & $\begin{array}{l}\text { Decisión } 578 \text { de } \\
2004 \text { CAN }\end{array}$ & $x X$ & $\begin{array}{c}\text { Dec Supr No } 297 \text { de } 2007 \text { (Chi) y Res } \\
\text { No } 27905 \text { de } 2004 \text { (Per) }\end{array}$ & $\begin{array}{l}\text { Dec Sup No 003- } \\
\text { 2014-RE (Per) }\end{array}$ \\
\hline Chile & $\begin{array}{l}\text { LEY } 1261 \text { de } \\
2008(\mathrm{Col} \text { ) y Dec } \\
\text { Supr No } 232 \\
\text { de2009 (Chi) }\end{array}$ & $\begin{array}{c}\text { Dec Supr No } 297 \text { de } 2007 \text { (Chi) y Res } \\
\text { No } 27905 \text { de } 2004 \text { (Per) }\end{array}$ & $\mathrm{XX}$ & $\begin{array}{c}\text { ORD. } \mathrm{N}^{\circ} 3.489, \mathrm{DE} \\
11.12 .2009)\end{array}$ \\
\hline México & $\begin{array}{l}\text { Ley } 1568 \mathrm{del} \\
2012\end{array}$ & Dec Sup Nº03-2014-RE (Per) & ORD. $N^{\circ} 3.489$, DE 11.12.2009) & $X X$ \\
\hline
\end{tabular}

Tabla 3 Convenios de no doble tributación. Fuente: Elaboración Propia.

Los tratados de no doble tributación son acuerdos internacionales que se suscriben entre dos o más países, para impedir que a un contribuyente se le atribuya varias veces gravámenes tributarios por un mismo hecho. No todos los acuerdos de no doble tributación son iguales, ya que unos acuerdos son aplicables a la totalidad de residentes del país, como es el caso de los acuerdos de los países el mercado integrado latinoamericano, y otros acuerdos son aplicado específicamente a ciertos sectores económicos (Tellez, 2016)

El primer convenio de no doble tributación se dio entre Perú y Colombia dentro del marco de la comunidad andina de naciones, abarca los residentes tanto de Colombia como de Perú se dio hacia el año 2014, el convenio entre Chile y Colombia de no doble tributación abarca para prevenir la evasión fiscal en relación con el impuesto a la renta y al patrimonio y su protocolo entre estos países. El convenio con México se dio a raíz de la Alianza del Pacifico y la creación del Mercado Integrado Latinoamericano para el año 2012.

\section{METODOLOGÍA}

El objetivo de la investigación es analizar la carga tributaria para los inversionistas dentro del mercado integrado latinoamericano y su efecto sobre la compra y venta de acciones. Se inicia con un análisis comparado de las tarifas de los impuestos de los diferentes países que integran el MILA. Como primer paso se analiza, mediante estudio descriptivo, la carga tributaria relacionada con cada uno de los impuestos asociados a la compra y venta de acciones en personas jurídicas, en los cuales el inversionista recibe rentas por concepto de dividendos y ganancias de capital.

En segunda instancia, con base en el análisis realizado se desarrolla un aplicativo en Excel con el cual se ejemplifica una inversión hipotética en los distintos mercados que conforman el MILA, en este supuesto, la investigación se centrara en los inversionistas reconocidos como "personas jurídicas", ya que son los inversores que más influyen en los mercados de valores en el mundo por el monto de sus inversiones, las empresas invierten grandes inyecciones de capital en el mercado accionario y en los mercados financieros en general, por estas razones las empresas son los inversionistas que le dan una mayor dinamismo al mercado accionario.

Con el desarrollo de la simulación en Excel se pretende visualizar los países con la mayor y menor carga tributaria en la compra y venta de acciones. El ejemplo realizado se desarrolla bajo los siguientes supuestos:

- La tasa de cambio es el promedio anual de cada país del año 2018, la tasa no tiene variación a lo largo de la inversión. 
- La inversión inicial se realiza con 100.000 dólares que se convierten a su equivalencia en la moneda de cada país.

- La comisión hipotética de transacción por compra de acciones estándar es del $0.70 \%$.

- La comisión hipotética de transacción por venta de acciones estándar es del $0.80 \%$.

- El periodo de tiempo para el cálculo de la inversión se realiza a un año (desde 02 de enero de 2018 hasta 31 de diciembre de 2018).

- El valor por acción se fija en 20.29 dólares.

- La rentabilidad estimada es del 15\% sobre el valor final de Venta del total de las acciones.

- El dividendo por acción es de 1.5usd estándar.

- Se estima que cada operación no superará el 7\% de las acciones en circulación de la empresa emisora.

\section{Desarrollo metodológico}

En los mercados de valores, más específicamente en las acciones se presentan dos maneras de obtener ganancias por parte de inversionistas. La primera se da cuando, el precio de venta es mayor al de compra. La segunda, cuando el accionista recibe los dividendos ocasionados por la distribución de utilidades que realizan las empresas generalmente cada año. Estas dos formas de obtener ganancias provocan que los inversionistas deban realizar los pagos fiscales por la enajenación de los productos financieros y por recibir una parte de las utilidades de una empresa. Añadido a esto, los contribuyentes en cada país que cumplan con ciertos requisitos están obligados a realizar declaración de renta anualmente por los ingresos que generaron en el año, así los inversionistas que obtengan buenas ganancias o beneficios obtenidos en operaciones de compra y venta de acciones deben declarar.

Teniendo esto en cuenta, los países integrantes del MILA tomaron medidas para evitar la doble tributación y prevenir la evasión de impuestos que se pueden producir en las operaciones comerciales de estos países. De ahí que sea importante describir las diversas tarifas tributarias que se dan con la compra y venta de acciones entre los países integrantes del MILA.

Para realizar una adecuada comparación de los impuestos mencionados se efectúa previamente una aclaración sobre los conceptos de ganancias de capital y ganancias por dividendos como se presenta a continuación. Un dividendo es un beneficio económico que reparte una compañía entre sus accionistas, de acuerdo a la participación de estos, sobre el capital social de la empresa, es un derecho económico del dueño de la acción (Ramirez, 2011). La repartición de los dividendos se puede dar de acuerdo con el ejercicio anual o semestral de la empresa, en conjunto con la política de repartición de dividendos que posee la empresa.

Para el desarrollo de la metodología se tuvo realizó una revisión de literatura y documentos Web como fuente principal de información las bolsas de valores de los países del MILA, la página principal de MILA, normatividad tributaria y datos tributarios relevantes, se tuvieron en cuenta estudios e investigaciones relacionados al propósito de la presente investigación. 
Análisis normativo

\begin{tabular}{|c|c|}
\hline \multicolumn{2}{|r|}{ Ganancias por Dividendos } \\
\hline Colombia & $\begin{array}{l}\text { Reglas aplicables a los dividendos que se repartan con cargo a utilidades generadas a partir del año gravable } 2017 \text {. Los } \\
\text { dividendos susceptibles de distribuirse como no gravados, a un inversionista de portafolio del exterior, están sujetos } \\
\text { a una tarifa de impuesto sobre renta del } 5 \% \text {. Los dividendos susceptibles de distribuirse como gravados, a un } \\
\text { inversionista de portafolio del exterior, están sujetos a una tarifa de impuesto sobre renta del } 25 \% \text {, más la tarifa del } \\
5 \% \text {, una vez disminuido el impuesto del } 25 \% \text {. Decreto } 2250 \text { de } 2017\end{array}$ \\
\hline Chile & $\begin{array}{l}\text { Tasa de retención del } 35 \% \text {, aplicable para personas jurídicas y naturales (Personas no residentes sin convenio } \\
\text { tributario). De acuerdo con la Ley N²0.780, del } 29 \text { de septiembre del } 2014 \text {. Los contribuyentes del impuesto adicional } \\
\text { que sean residentes de en un país (CDTI) y esté vigente, se acuerda que respecto al impuesto adicional pueda } \\
\text { aplicarse deducción completa del impuesto de Primera Categoría como crédito o alguna norma que otorgue el mismo } \\
\text { efecto tributario. En otras palabras, se pueda imputar el } 10 \% \text { del crédito del impuesto de Primera Categoría contra el } \\
\text { impuesto adicional en virtud del CDTI (suscrito y vigente) entre Chile y el país de residencia del contribuyente, tal } \\
\text { como lo establece el artículo } 63 \text { inciso tercero parte final LIR. }\end{array}$ \\
\hline México & $\begin{array}{l}\text { Retención definitiva 10\% (tasa según CDI del 0\% hasta 10\%). Aplicable para personas jurídicas y naturales. Resolución } \\
\text { Miscelánea } 2015 .\end{array}$ \\
\hline Perú & $\begin{array}{l}\text { Retención en la fuente de los dividendos percibidos, } 2017 \text { - 2018: 8.0\% y } 2019 \text { en adelante: 9.3\%. Aplicable para } \\
\text { personas jurídicas y naturales (no domiciliados). Tasas señaladas en la Ley N³0296 }\end{array}$ \\
\hline
\end{tabular}

Tabla 4. Impuestos sobre ganancias por dividendos por país. Fuente: (MILA Mercado Integrado Latinoamericano, 2019)

Para el año 2018, el impuesto de dividendos en Colombia está en el 30\%. Esto implica que un inversionista de portafolio del exterior está sujeto a una tarifa de impuesto sobre renta del $25 \%$, más la tarifa del $5 \%$, y para el mercado chileno se encuentra a una tasa de retención del 35\%, Para el caso de Perú, la tasa el del $8 \%$, para el año 2018, así como del 10\% para el caso de México. De esta forma los inversionistas del MILA ven como una clara ventaja competitiva a los países que tengan unas tasas de retención más bajas sobre dividendos, ya que esto es importante sobre todo para los inversionistas de largo plazo.

De acuerdo a las tasas de retención de los diferentes tributos de los países, sin duda las ganancias por dividendos que pagan un menor valor de impuestos, en comparación con los otros tres países, son los dividendos de las empresas del mercado Peruano, seguido de las empresas del mercado Mexicano, por esta razón, estos dos países son los que cuentan con las tasas de retención más bajas, entre los países que integran el MILA. De este modo, no se observa relación entre la tarifa impositiva de dividendos y la integración de los mercados, una muestra de esta situación es que el mercado peruano tiene la tarifa impositiva más baja y aun así es el mercado con menor participación en el MILA.

\begin{tabular}{|ll|}
\hline Colombia & \multicolumn{1}{c|}{ Ganancias por capital } \\
\hline & $\begin{array}{l}\text { Tasa del 0\% si la venta no supera el 10\% de las acciones en circulación de la emisora en el mismo periodo fiscal. 14\% de } \\
\text { retención sobre el valor de la venta si esta supera el 10\% de las acciones en circulación de la emisora. Si vende más del } \\
10 \% \text { de las acciones en circulación, se debe presentar una declaración de renta pagando un impuesto de 10\% (si la } \\
\text { acción fue poseída por un periodo superior a } 2 \text { años o del 17\% (si los poseía por menos de dos años) sobre el valor de } \\
\text { la utilidad (precio de venta menos costo). El valor que le haya retenido lo podrá restar del monto a pagar de } \\
\text { impuesto. art. 18-1 ET y articulo } 56 \text { de la ley 1819 }\end{array}$ \\
Chile & $\begin{array}{l}\text { 0\% cuando se trate de enajenación de acciones que cumplan los requisitos del art. 107 LIR y para acciones del } \\
\text { segmento emergente. 35\% cuando se trate de acciones sin presencia bursátil. Aplicable para personas jurídicas y } \\
\text { naturales. }\end{array}$
\end{tabular}




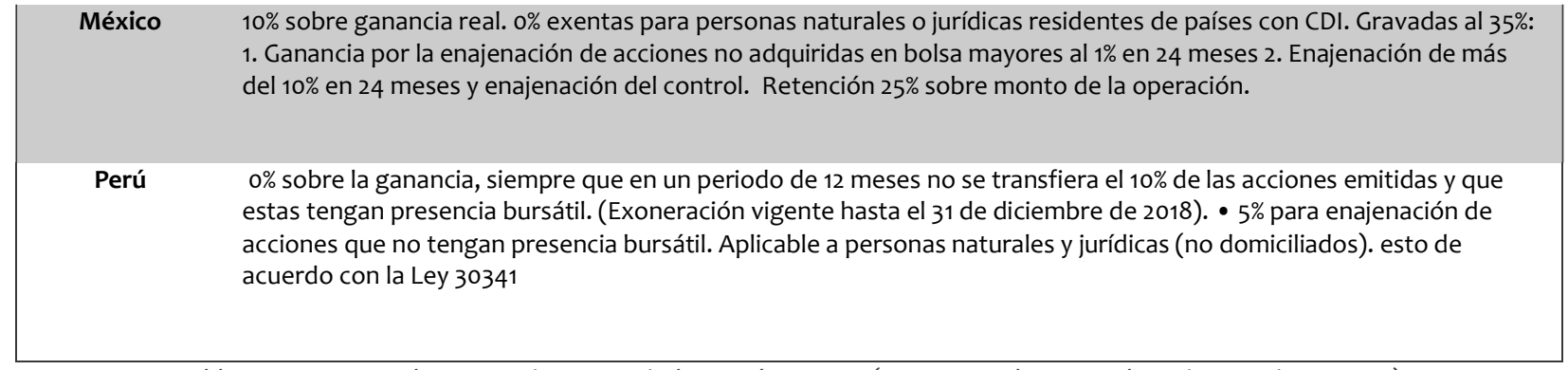

Tabla 5. Impuestos sobre ganancias por capital por país. Fuente: (MILA Mercado Integrado Latinoamericano, 2019)

En cuanto al concepto de ganancias de capital se refiere al beneficio que se obtiene por la venta de un activo financiero, teniendo en cuenta que el precio de venta sea mayor al precio de compra, en este caso se obtiene una ganancia de capital, si se da el caso contrario se daría una pérdida del capital (Duque J. , 2017).

Sin embargo, se debe también analizar el caso cuando se reciben ingresos por ganancias de capital, que básicamente es la diferente entre el precio de adquisición y venta de un activo financiero, en este caso una acción, ya que cuando las ganancias son por aumento en el precio de la acción y luego, cuando el inversionista vende las acciones, los impuestos en Colombia son del o\% de retención si la venta no supera el 10\% de las acciones en circulación de la emisora en el mismo periodo fiscal, $14 \%$ de retención sobre el valor de la venta si esta supera el 10\% de las acciones en circulación de la emisora.

Para el caso de Chile, para personas jurídicas y naturales se les aplica una tasa de retención del 0\%, para Perú 0\% sobre la ganancia, siempre que en un periodo de 12 meses no se transfiera el $10 \%$ de las acciones emitidas y que estas tengan presencia bursátil y $5 \%$ para enajenación de acciones que no tengan presencia bursátil aplicable a personas naturales y jurídicas (no domiciliados) y por ultimo México con $0 \%$ exentas para personas naturales o jurídicas residentes de países con CDI o Convenios de doble imposición (MILA Mercado Integrado Latinoamericano, 2019).

Colombia ha perdido atractivo hacia los inversionistas, debido a las continuas reformas tributarias en los últimos años, este es un aspecto muy importante y se convierte en un argumento a favor de la simplificación del sistema tributario para poder lograr un funcionamiento más eficiente y consistente, que genere ventajas competitivas frente a los países de la región, lo cual generar una mayor confianza para los inversionistas.

Un inversionista que invierte en el mercado MILA, no solo debe considerar las tarifas de los impuestos en cada país integrante del MILA, ya que al obtener muy buenas ganancias sobre activos financieros en este caso acciones de algún país del MILA, además el inversionista tiene que declarar renta. Asímismo, se debe tener en cuenta los convenios de doble tributación existentes o también llamados (CDI), si se tienen deducciones para disminuir la declaración de renta que son permitidas en cada país, así como los incentivos, descuentos y tiempos de pago de la declaración de renta, es preciso conocer por parte del inversionista si se puede diferir el impuesto o no, como se evidencia son diversos los factores que un inversionista deben tener presente, y que para una mayor certeza por parte del inversionista, sería una buena opción un mayor asesoramiento tributario, para saber dependiendo de los montos de ganancias obtenidos, ya sea por causa de dividendos o ganancias de capital, la mejor manera de manejarlo, para no pagar un valor elevado de impuesto de renta.

Para Perú y Colombia la tasa de retención es del o\%, siempre y cuando el inversionista no tenga ninguna venta o transferencia de más del $10 \%$ de las acciones en circulación de una empresa emisora, para el caso de Colombia si se vende más del 10\% de las acciones en circulación, se debe presentar una declaración de renta pagando un impuesto de 10\% (si la acción fue poseída por un periodo superior a 2 años o del 17\%, si los poseía por menos de dos años). Para el caso de Perú 5\% para enajenación de acciones que no tengan presencia bursátil. 
Las tarifas que presenta México son Tarifas 10\% sobre ganancia real, o\% exentas para personas naturales o jurídicas residentes de países con CDI. Gravadas al 35\%: 1. Ganancia por la enajenación de acciones no adquiridas en bolsa mayores al 1\% en 24 meses 2. Enajenación de más del 10\% en 24 meses y enajenación del control, retención $25 \%$ sobre monto de la operación. Por otro lado, chile $0 \%$ cuando se trate de enajenación de acciones que cumplan los requisitos del art. 107 LIR y para acciones del segmento emergente. 35\% cuando se trate de acciones sin presencia bursátil, siendo así, aunque todos los países tienen una tarifa del 0\% si no superan en comprar más del 10\% de acciones en circulación de una empresa emisora, para montos mayores el país con menor tasa de retención seria Perú, y México podría a tener una retención hasta del 25\% por un volumen elevado de acciones y que se administren en el portafolio por un periodo superior a más de 24 meses.

Lo anterior indica que no hay una relación directa entre las asimetrías tributarias respecto de los volúmenes negociados y la liquidez del mismo, ya que Perú tiene la tasa de retención más baja respecto a los otros países que confirman el mercado, no obstante, los volúmenes de negociación de Perú son menores respecto a los demás países integrantes del MILA.

\section{Análisis numérico}

Para cumplir con el objetivo de la investigación se realizó una simulación de compra que se presenta a continuación:

\begin{tabular}{|c|c|c|c|}
\hline Casilla & Simulación Compra Colombia & Variables Metodología Estándar & Monto \\
\hline \multirow[t]{2}{*}{1} & Inversión (Compra) en USD & & \\
\hline & & & $100.000,00$ \\
\hline \multirow[t]{2}{*}{2} & Tasa de Cambio Prom 2018 (COP) & tasa promedio año 2018 TRM & \\
\hline & & & $2.956,41$ \\
\hline \multirow[t]{2}{*}{3} & Equivalencia Inversión| en moneda local & Casilla $1 \times 2$ & \\
\hline & & & $295.641 .101,37$ \\
\hline 4 & Precio por acción Hipotética de compra & 20,29 usd & $\begin{array}{c}\$ \\
59.985,58\end{array}$ \\
\hline 5 & $\begin{array}{l}\text { Número de acciones equivalentes al mercado } \\
\text { local }\end{array}$ & Casilla 6 / casilla 4 & $4.894,036$ \\
\hline \multirow[t]{2}{*}{6} & Inversión en Moneda local & Casilla 3 - Casilla 7 & \\
\hline & & & $293.571 .613,66$ \\
\hline \multirow[t]{2}{*}{7} & (+) Comisión de transacción(compra) & $0,7 \%$ se aplica a la casilla 3 & \\
\hline & & & $2.069 \cdot 487,71$ \\
\hline \multirow[t]{2}{*}{8} & (+)Impuestos de Compra(si aplica) & $\mathrm{N} / \mathrm{A}$ & \\
\hline & & & - \\
\hline 9 & (=)Total Egresos Compra & Suma Casilla $5+6+7$ & $295.641 .101,37$ \\
\hline \multirow[t]{2}{*}{10} & Ajuste saldo a favor en compra & Casilla 3 - Casilla 9 & \\
\hline & & & - \\
\hline 11 & Simulación Dividendos & Variables Metodología Estándar & Monto \\
\hline \multirow[t]{2}{*}{12} & Dividendos Persona Jurídica (USD) & & \\
\hline & & & $5.505,79$ \\
\hline \multirow[t]{2}{*}{13} & Otros ingresos & & \\
\hline & & & - \\
\hline \multirow[t]{2}{*}{14} & Dividendos Anuales usd & $1,5 \%$ rendimiento por casilla 5 & \\
\hline & & & $7.341,05$ \\
\hline \multirow[t]{2}{*}{15} & (=)Dinero Neto por Dividendos Anuales (COP) & Casilla $14 \times$ casilla 2 & \\
\hline & & & $21.703 .174,99$ \\
\hline 16 & $\begin{array}{c}\text { (-) Tarifa de Impuesto asociados a los } \\
\text { dividendos }\end{array}$ & Depende la tarifa de impuesto y si aplica & $5.425 \cdot 793,75$ \\
\hline 17 & (=)Dinero neto recibido por Dividendos & Casilla 15 - Casilla 16 & \\
\hline
\end{tabular}


Para la réplica de este ejercicio es fundamental tener presente las tarifas que aplican y no aplican dependiendo el monto de la inversión en el año, tener claro qué tipo de régimen al que pertenece el inversionista, si es persona natural o jurídica, así como el listado de los anteriores supuestos mencionados, y teniendo esta información y datos establecidos se realizan varios análisis en gráficos comparados acerca de las tarifas de los impuestos relacionadas con cada uno de los impuestos asociados a la compra y venta de acciones en personas jurídicas. Ya que son los inversores que más influyen en los mercados de valores en el mundo, por el monto de sus inversiones y de acuerdo con esto se analiza en qué país el inversionista tiene la inversión más rentable, y si esto tiene una relación con variables como el monto de negociación de cada país dentro del MILA, o el número de emisores de cada país dentro del MILA.

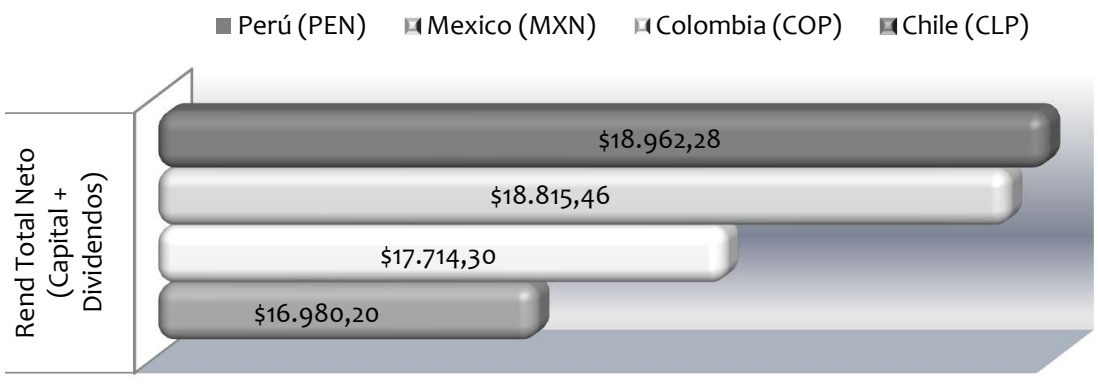

Figura 2 Rendimientos Netos Totales (Capital \& Dividendos en Usd). Fuente: Elaboración propia

Según los resultados en cuanto a utilidades por conceptos de capital y dividendos, se evidencia que Perú obtiene la mayor rentabilidad Neta según la simulación establecida en un monto de 18.962.28 USD, seguido muy de cerca por México con una rentabilidad neta de 18.815.46 USD, en tercer lugar, se encuentra Colombia con una rentabilidad Neta de 17.714.30 USD y por último, Chile con una rentabilidad neta de16.980.20 USD. Esto establece una diferencia asimétrica en rentabilidad para un inversionista en cuanto a la optimización del portafolio dadas las tarifas impositivas de los países que conforman el MILA.

En términos de utilidades por conceptos de capital y dividendos (ver figura 3), se evidencia que Perú obtiene el mayor porcentaje de rentabilidad Neta según la simulación con un $26.16 \%$ frente a las rentabilidades de todos los países, seguido muy de cerca por México con una rentabilidad del $25.96 \%$, en tercer lugar, se encuentra Colombia con una rentabilidad del $24.44 \%$ y por último Chile con una rentabilidad del $23.43 \%$. Con esto se puede observar que los países del MILA, tienen una diferencia asimétrica en rentabilidad para un inversionista. De acuerdo con estas cifras la carga tributaria de un país, no necesariamente tiene una repercusión directa sobre el volúmenes de transacciones sobre las acciones de las empresas de este país, pues Perú tiene la carga más baja con el menor volumen de negociaciones mientras Chile que es un país con una de las tasas de impuestos más altas, tiene volúmenes de negociación mucho mayores, por lo cual la carga tributaria no es una barrera para la integración de los mercados. 
- Colombia (COP) $\quad$ Chile (CLP) $\square$ Mexico (MXN) $\square$ Perú (PEN)

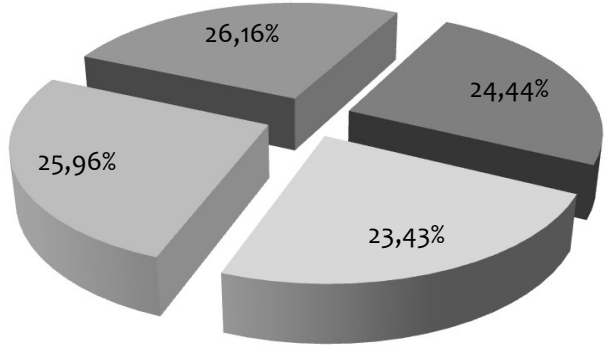

Figura 3 Rendimientos Netos Totales (Capital \& Dividendos en \%). Fuente: Elaboración propia

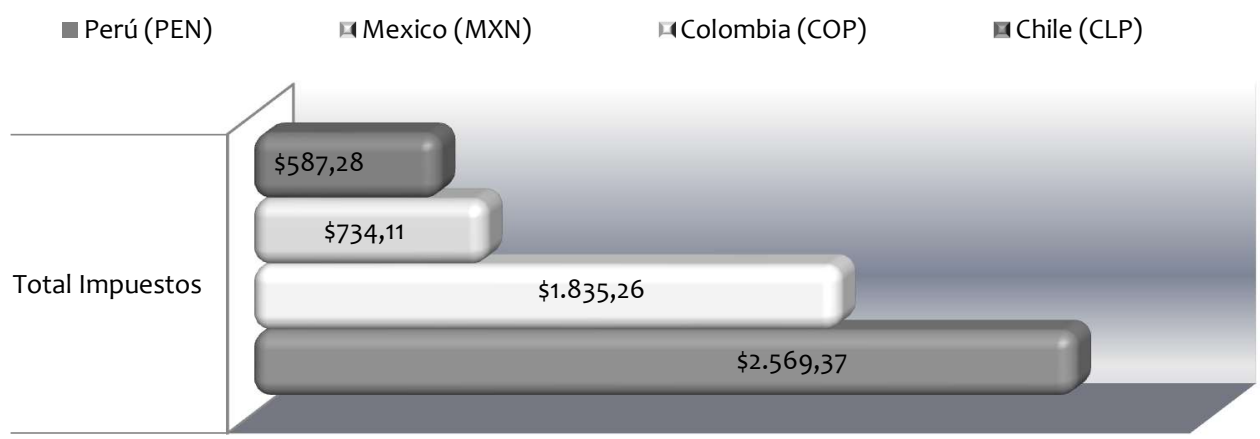

Figura 4 Impuestos Causados (Dividendos en Usd). Fuente: Elaboración propia

Se observa en la figura 4, de acuerdo a los resultados en cuanto a concepto de impuestos causados por dividendos, los países con mayor valor de impuestos causados expresados en USD son Chile con 2569.37 USD, seguido en segundo lugar por Colombia con 1835.26 USD, para el tercer lugar se encuentra México con 734.11 USD y en cuarto lugar Perú con 587.28 USD, como se puede observar entre Perú y México tienen montos similares por concepto de impuestos, pero para Colombia y Chile que ocupan el tercero y cuarto lugar, se puede observar una diferencia considerable en cuanto al valor de los impuestos causados.

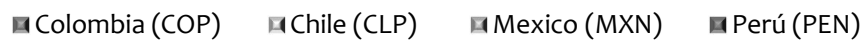

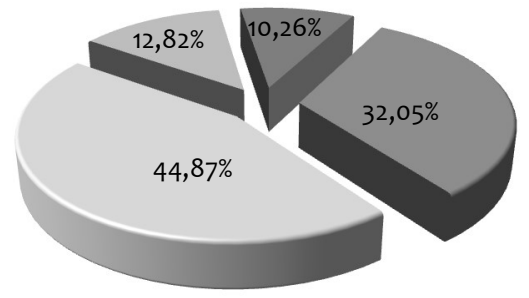

Figura 5. Impuestos Causados (Dividendos en \%). Fuente: Elaboración propia 
De acuerdo a la figura 5, en cuanto a los resultados por concepto de impuestos sobre dividendos, los países con mayor peso porcentual de impuestos frente al ejercicio simulado son Chile con un $44.87 \%$, seguido en segundo lugar por Colombia con un 32.05\%, para el tercer lugar se encuentra México con un 12.82\% y en cuarto lugar Perú con un $10.26 \%$, como se observa, los países con mayor porcentaje de impuestos causados son Chile y Colombia ya que poseen mayores tasas porcentuales en comparación a los países de México y Perú.

\section{CONCLUSIONES}

Con la realización de la presente y usando el desarrollo de un aplicativo en Excel se encontró que las tasas de retención por dividendos que pagan un menor valor de impuestos, son los dividendos de las empresas del mercado peruano, seguido de las empresas del mercado mexicano, siendo la tarifa de México un $2 \%$ más alta que la de Perú, estos dos países tienen las tasas de retención más bajas, seguidos de Colombia y en último lugar Chile que es el país con la mayor tasa de retención.

En cuanto a los ingresos por ganancias de capital, las tasas de retención de los países del MILA son del 0\% para el ejercicio simulado, por ende, se le da un mayor valor en cuanto a carga tributaria a las tasas sobre dividendos de los países del MILA. México posee la mayor capitalización bursátil con un $45 \%$ de la capitalización del MILA para el año 2016 y 2017, Perú con un 16\% y 11\% para el 2016 y 2017 respectivamente, Colombia con un 13\% para el 2016 y 2017. Del volumen de negociación del MILA en el 2018 México representa un 61\%, seguido por Chile con un 29\%, en tercer lugar, Colombia con un $8 \%$ y por ultimo Perú con un $2 \%$, las acciones de las empresas con mayor volumen de negociación durante el año 2018, son Falabella de Chile en primer lugar, en segundo lugar está la empresa Naf Trac de México, en tercer lugar se encuentra la empresa Ecopetrol, y finalmente el Banco de Crédito del Perú, el mercado de valores colombiano es el mas pequeño en lo que respecta al numero de emisores que lo conforman.

En contraste, el mercado de mayor tamaño por numero de emisores es el mercado peruano, se puede entonces concluir que aunque peru posee la tasa de retencion mas baja, su volumen de negociacion tambien es el mas bajo de los paises del MILA, asi como la capitalizacion bursátil y su acción mas negociada fue la del Banco de Crédito del Perú, que tuvo el valor de negociación más bajo, en caso contrario, Chile que tiene la tasa por impuesto más alta, tuvo la acción más negociada durante el 2018 en el MILA con la empresa Falabella, así como tiene un volumen de negociación y de capitalización bursátil superior en gran magnitud al de Perú. El número de emisores no es un indicador de tamaño de la negociación, ya que Perú es el que tiene mayor número de emisores, sin embargo, es el que cuenta con menores volúmenes de negociación (MILA Mercado Integrado Latinoamericano, 2018).

Según los economistas (Modigliani \& Merton, 1958) una de las mayores imperfecciones de los mercados financieros está relacionados con la existencia de impuestos, así como costos transaccionales y la asimetría informativa, entre otros. Los resultados del MILA muestran que la diferencia en las tasas de impuestos no son determinastes directos en cuanto al volumen de negociacion, la capitalizacion bursatil y las acciones mas negociadas. De los datos obtenidos mediante el simulador en Excel, las utilidades por conceptos de capital y dividendos, Perú obtiene la mayor rentabilidad neta con un monto de 18.962.28 USD, seguido muy de cerca por México con una rentabilidad neta de 18.815.46 USD, en tercer lugar, se encuentra Colombia con una rentabilidad Neta de 17.714.30 USD y por último, Chile con una rentabilidad neta de16.980.20 USD. Esto establece una diferencia asimétrica en rentabilidad para un inversionista en cuanto a la optimización del portafolio dadas las tarifas impositivas de los países que conforman el MILA con estos datos se muestra que la carga tributaria no es una barrera para la integración de los mercados.

Los países con mayor valor de impuestos causados expresados en USD son Chile con 2569.37 USD, seguido en segundo lugar por Colombia con 1835.26 USD, para el tercer lugar se encuentra México con 734.11 USD y en cuarto lugar Perú con 587.28 USD, con esto se ve que Perú y México tienen montos similares por concepto de impuestos, 
pero para Colombia y Chile que ocupan el tercero y cuarto lugar, se puede observar una diferencia considerable en cuanto al valor de los impuestos causados.

Para finalizar un inversionista que invierte en el mercado MILA, no solo debe considerar las tarifas de los impuestos de cada país, se pueden obtener muy buenas ganancias sobre activos financieros en este caso acciones, en el caso de que el inversionista tenga que declarar renta, y para su declaración deberá tener en cuenta factores como, los convenios de doble tributación existentes o también llamados (CDI), si se tienen deducciones para disminuir el valor a pagar de la declaración de renta de acuerdo a cada país, así como los incentivos, descuentos y tiempos de pago de la declaración de renta, es preciso conocer por parte del inversionista si se puede diferir el impuesto o no, son varios factores que un inversionista deben tener presente, y que para una mayor certeza por parte del inversionista, sería una buena opción un mayor asesoramiento tributario, para no pagar un valor elevado de impuesto de renta. Ya que no siempre por un país tener la tasa de impuestos más alta quiere decir que pague un mayor impuesto, o por un país tener una tasa de impuestos más baja, no significa que será el que más transacciones y volúmenes de negociación tenga sobre las acciones de las empresas de su país como se evidencia en la presente investigación.

\section{REFERENCIAS BIBLIOGRÁFICAS}

Academia de Inversion. (08 de Septiembre de 2013). Capitalización bursátil: Definición, clasificación, cálculo y ejemplos. Recuperado el 04 de Julio de 2019, de Academia de Inversion: https://www.academiadeinversion.com/capitalizacion-bursatil-definicion-clasificacion-calculo-yejemplos/

Almazan, N. (17 de Junio de 2015). ICTSD. Obtenido de MILA, logros y desafíos de un mercado integrado regional: https://es.ictsd.org/bridges-news/puentes/news/mila-logros-y-desaf\%C3\%ADos-de-un-mercadointegrado-regional

Almazan, N. (17 de Junio de 2015). MILA, logros y desafíos de un mercado integrado regional. Recuperado el 27 de Febrero de 2019, de ICTSD: https://es.ictsd.org/bridges-news/puentes/news/mila-logros-ydesaf\%C3\%ADos-de-un-mercado-integrado-regional

Arboleda, M., Valencia, M., \& Hernandez, P. (s.f. de s.f. de 2012). IMPACTO EN EL MERCADO DE ACCIONES COLOMBIANO CON LA IMPLEMENTACIÓN DEL MILA. Recuperado el 19 de Febrero de 2019, de Universidad de Medellin: https://repository.udem.edu.co/bitstream/handle/11407/266/Impacto\%20en\%20el\%2omercado\%20de\%20 acciones\%20colombiano\%20con\%20la\%20implementaci\%C3\%B3n\%20del\%20MILA.pdf?sequence=1

Azofra, V., \& Lopez, F. (s.f. de s.f. de 2000). L a asimetria informatica en los mercados financieros: ¿El hallazgo de un nexo de union? Recuperado el 17 de Abril de 2019, de Dialnet: file:///C:/Users/john\%2olancheros/Downloads/DialnetLaAsimetrialnformativaEnLosMercadosFinancieros-785012\%20(1).pdf

Banco Nacional de Mexico. (s.f. de s.f. de s.f.). Manejo de Riesgo - La Teoría Moderna de Portafolios. Recuperado el 10 de Julio de 2019, de Banco Nacional de Mexico: https://www.bancanetempresarial.banamex.com.mx/spanishdir/tutorial/spanishdir/Teoria_Moderna_P ortafolios.htm 
Cabieses, G. (12 de Noviembre de 2013). Fama y los mercados eficientes. Recuperado el 07 de Julio de 2019, de elcato: https://www.elcato.org/fama-y-los-mercados-eficientes

Comision Economica para America Latina. (s.f. de s.f. de 2016). AMÉRICA LATINA (14 PAÍSES): PRINCIPALES MEDIDAS Y REFORMAS TRIBUTARIAS, AÑO 2016. Recuperado el 08 de Abril de 2019, de Comision Economica para America

Latina: https://repositorio.cepal.org/bitstream/handle/11362/41044/7/PF2017Cuadros_analiticos.pdf

Decreto Supremo N 133-2013-EF. (31 de Diciembre de 2016). Ministerio de Economía y Finanzas. Decreto Supremo Que Aprueba El Texto Unico Ordenado Del Codigo Tributario. Peru, Peru. Recuperado el 19 de Mayo de 2019, de https://www.grupoconsultorefe.com/assets/files/recursos/files/Per\%C3\%BA\%20\%20C\%C3\%B3digo\%20Tributario_4007.pdf

Duque , E., \& Ramirez, J. (s.f. de s.f. de 2013). Análisis del impacto generado a raíz del acuerdo MILA en el volumen transaccional del Mercado Accionario Colombiano en el período 2010 - 2013, aplicando el Test de Chow. Recuperado el 25 de Febrero de 2019, de Institución Universitaria Esumer: revistas.esumer.edu.co/index.php/merc/article/download/91/105

Duque, J. (24 de Noviembre de 2017). ¿Qué es una ganancia de capital? Recuperado el 20 de Abril de 2019, de ABC Finanzas: https://www.abcfinanzas.com/administracion-financiera/que-es-una-ganancia-de-capital

Franco, L., Avendaño, C., \& Barbutin, H. (s.f. de Junio de 2011). Modelo de Markowitz y Modelo de Black-Litterman en la Optimización de Portafolios de Inversión. Recuperado el 10 de Julio de 2019, de Revista Scielo: http://www.scielo.org.co/scielo.php?script=sci_arttext\&pid=S0123-77992011000100005

Hyme, P. (s.f. de s.f. de 2013). LA TEORÍA DE LOS MERCADOS DE CAPITALES EFICIENTES. UN EXAMEN CRÍTICO. Recuperado el 17 de Marzo de 2019, de Revista Scielo: http://www.scielo.org.co/scielo.php?script=sci_arttext\&pid=S0121-47722003000200004

Jimenez, J. (27 de 09 de 2013). Inversion Finanzas. Obtenido de Bolsa para novatos: Seis teorías del mercado que debes conocer: http://www.finanzas.com/noticias/mercados/bolsas/20130927/bolsa-para-novatos-seis2491303.html

Lancheros, J. (s.f. de Junio de 2017). ANALISIS DEL PROCESO DE INTEGRACION DEL MERCADO LATINOAMERICANO (MILA) DESDE EL 2011 AL 2016. Recuperado el 04 de Julio de 2019, de Corporacion Universitaria Iberoamericana:

http://repositorio.iberoamericana.edu.co/bitstream/001/414/1/An\%C3\%A1lisis\%20del\%2oproceso\%20de\%20 integraci\%C3\%B3n\%20del\%2omercado\%2olatinoamericano\%20\%28MILA\%29\%20desde\%20el\%202011\%20al\%2 02016.pdf

Ley 1819. (29 de Diciembre de 2016). Secretaria del Senado. Por medio de la cual se adopta una reforma tributaria estructural. Colombia, Colombia. Recuperado el 19 de Mayo de 2019, de http://www.secretariasenado.gov.co/senado/basedoc/ley_1819_2016.html

Ley General de Sociedades Mercantiles. (14 de Junio de 2018). Camara De Diputados Del H. Congreso de la Union. De la constitución y funcionamiento de las Sociedades en general. Colombia, Colombia. Recuperado el 19 de Mayo de 2019, de http://www.diputados.gob.mx/LeyesBiblio/pdf/144_140618.pdf

Ley Numero 20.956. (26 de Octubre de 2016). Ministerio de Hacienda. Establece Medidas Para Impulsar La Productividad. Chile, Chile. Recuperado el 19 de Mayo de 2019, de https://www.leychile.cl/Navegar?idNorma=1095967 
Libre, D. (11 de Noviembre de 2013). La importancia del Mercado de Valores. Recuperado el 26 de Febrero de 2019, de Diario Libre: https://www.diariolibre.com/opinion/la-importancia-del-mercado-de-valoresENdl410895

MILA Mercado Integrado Latinoamericano. (31 de Diciembre de 2018). Datos Mercado MILA. Recuperado el 04 de Julio de 2019, de MILA Mercado Integrado Latinoamericano: http://mercadomila.com/?s=capitalizacion\&lang=es

MILA Mercado Integrado Latinoamericano. (s,f, de s,f, de 2019). Informacion Tribibutari MILA. Recuperado el 07 de Julio de 2019, de Mercado MILA: https://mercadomila.com/wpcontent/uploads/2018/04/MILA_Info_Tributaria.pdf

MILA Mercado Integrado Latinoamericano. (s.f.). Reseña Historica. Obtenido de MILA Mercado Integrado Latinoamericano.

Mila. (s.f. de s.f. de s.f.). Reseña Historica. Recuperado el 04 de Julio de 2019, de Mercado MILA: https://mercadomila.com/quienes-somos/resena-historica/

Modigliani, F., \& Merton, M. (s.f. de s.f. de 1958). Teoria de las Finanzas Corporativas. Teoría de las Finanzas Corporativas, pág. s.f. Recuperado el 24 de Abril de 2019

Modigliani, F., \& Miller, M. (s.f. de s.f. de 1963). Teorema de Modigliani y Miller. Teorema de Modigliani y Miller, pág. s.f. Recuperado el 06 de Julio de 2019

Pinilla, C. (2014). Colegio de Estudios Superiores de Administracion. Obtenido de EL PAPEL DEL MERCADO BURSATIL EN EL CICLO ECONOMICO COLOMBIANO: http://repository.cesa.edu.co/bitstream/handle/10726/1422/TMM00313.pdf?sequence=1\&isAllowed=y

Pinilla, C., \& Pineda, J. (s.f. de s.f. de 2011). CRECIMIENTO Y FINANZAS: EL PAPEL DEL MERCADO BURSÁTIL EN COLOMBIA. Recuperado el 17 de Abril de 2019, de Universidad de ls Salle: http://repository.lasalle.edu.co/bitstream/handle/10185/12495/T10.11\%20P655c.pdf;jsessionid=20156EA9 ED2911F983EFEA8763B2984A?sequence=1

Prieto, D., \& Fajardo, W. (s.f. de Junio de 2015). LA INTEGRACIÓN DE LOS MERCADOS DE VALORES COMO MECANISMO DE CRECIMIENTO ECONÓMICO: CASO MERCADO INTEGRADO LATINOAMERICANO (MILA). Recuperado el 15 de Abril de 2019, de Universidad de la Salle: http://repository.lasalle.edu.co/bitstream/handle/10185/17467/63102146_2015.pdf

Ramirez, A. (s.f. de s.f. de 2011). De las utilidades a los dividendos por accion. Recuperado el 20 de Abril de 2019, de Universidad Javeriana: https://www.javeriana.edu.co/personales/hbermude/Audire/arr.pdf

Revista Dinero. (11 de Octubre de 2015). Estudiamos acuerdo de doble tributación en Alianza Pacífico -URF. Recuperado el 20 de Febrero de 2019, de Revista Dinero: https://www.dinero.com/inversionistas/articulo/estudiamos-acuerdo-doble-tributacion-alianza-pacificourf/215802

Revista Dinero. (15 de 02 de 2018). Dinero. Obtenido de Los emisores más destacados en la Bolsa de Valores de Colombia: https://www.dinero.com/edicion-impresa/negocios/articulo/emisores-mas-relevantes-enbolsa-de-valores-de-colombia/255492 
Revista Dinero. (04 de 12 de 2018). Dinero. Obtenido de Así fueron los resultados de las empresas que cotizan en bolsa en 2017: https://www.dinero.com/edicion-impresa/negocios/articulo/balances-2017-de-lasempresas-que-cotizan-en-bolsa-en-colombia/257206

Reyes, J. (03 de Abril de 2013). La relación entre crecimiento economico y el mercado bursatil. Recuperado el 17 de Abril de 2019, de Fundacion Dialnet: https://dialnet.unirioja.es/descarga/articulo/5061186.pdf

Rivera, J. (s.f. de Septiembre de 2002). TEORÍA SOBRE LA ESTRUCTURA DE CAPITAL. Recuperado el 07 de Julio de 2019, de Revista Scielo: http://www.scielo.org.co/scielo.php?script=sci_arttext\&pid=S012359232002000300002

Romero, Y., Ramirez, F., \& Guzman, D. (2013). Universidad Javeriana. Obtenido de Mercado Integrado Latinoamericano (MILA) análisis de correlación y diversificación de los portafolios de acciones de los tres países miembros en el 2007-2012: revistas.javeriana.edu.co/index.php/cuacont/article/view/6067/4888

Saenz, F. (02 de Diciembre de 2013). Efectos fiscales MILA. Recuperado el 18 de Febrero de 2019, de Rankia: https://www.rankia.cl/blog/analisis-ipsa/2056265-efectos-fiscales-mila

Tellez, G. (o9 de Octubre de 2016). Convenios para evitar la doble imposición aplicables en Colombia. Recuperado el 25 de Abril de 2019, de Gerencie: https://www.gerencie.com/convenios-para-evitar-la-dobleimposicion-aplicables-en-colombia.html

Universidad de Oviedo. (s.f. de s.f. de s.f.). Teorías sobre la decisión de dividendos en la empresa. Recuperado el 18 de Marzo de 2019, de Universidad de Oviedo: https://www.unioviedo.es/fgascon/DF/T6_Teorias_sobre_decision_de_dividendos

Uribe, J., \& Ulloa, I. (s.f. de Diciembre de 2011). REVISANDO LA HIPÓTESIS DE LOS MERCADOS EFICIENTES: NUEVOS DATOS, NUEVAS CRISIS Y NUEVAS ESTIMACIONES. Recuperado el o9 de Julio de 2019, de Revista Scielo: http://www.scielo.org.co/scielo.php?script=sci_arttext\&pid=S0121-47722011000200007

Vargas, W., \& Bayardo, J. (s.f. de Junio de 2013). EL MILA. MERCADO DE INTEGRACIÓN ENTRE CHILE, PERÚ Y COLOMBIA. Recuperado el 19 de Febrero de 2019, de Revista Scielo: http://www.scielo.org.co/scielo.php?script=sci_arttext\&pid=S1909-30632013000100006\#n_a1 